\title{
Effect of Statcom on Distance Relay Performance in a Transmission Line
}

\author{
Sriteja Alapati and B. Durga prasad
}

\begin{abstract}
This paper describes modelling of impedance protection relays with the STATCOM, and simulation results for investigating the operation of impedance-based protection relays in a power system containing a flexible alternating current transmission (FACTS) controller such as the static synchronous compensator (STATCOM). The analyses are modelling the STATCOM and the protection relays. The impact of the FACTS device on protection relays is studied using digital distance relay algorithm in MATLAB/SIMULINK for normal operating conditions as well as for fault conditions.
\end{abstract}

Index Terms-Distance relay, FACTS devices, power system protection, modeling, STATCOM, digital distance relay algorithm.

\section{INTRODUCTION}

The excellent advantages that MATLAB/ SIMULINK [1] has make MATLAB/SIMULINK a convenient and interactive tool for both numerous analysis and direct communications with relay's test program. This project describes how to use MATLAB/SIMULINK for automatic, interactive, and high performance testing relay system. Some examples and simulation results are also provided in this paper.

Traditional updating of a transmission system by constructing new transmission lines becomes extremely difficult because of economic and environmental pressures. High efficiency in terms of better utilization of existing transmission lines, without compromising on the quality and reliability of electrical power apply has thus to be found via alternative means. In this respect, due to the recent advances in high power semiconductor technology, Flexible AC transmission System (FACTS) technology has been proposed to solve this problem. However, because of the added complexity due to the interaction of FACTS devices with the transmission system, the transients superimposed on the power frequency voltage and current waveforms (particularly under faults) can be significantly different from those systems not employing FACTS devices and it will result in rapid changes in system parameters such as line impedance and power angle. It is thus vitally important to study the impact of the FACTS devices on the traditional protection relay scheme such as the impedance-based distance protection relay.

statcom is one of the most widely used FACTS devices. It is based on a voltage source convert and can inject an

Manuscript received June 17, 2012; revised July 30, 2012

The authors are with GITAM university Vishakapatnam, India (e-mail: sritejaalapati@gmail.com,durga206@gmail.com). almost sinusoidal current with variable magnitude and in quadrature with the connecting line voltage .It is widely used at area to maintain the connecting point voltage by supplying or absorbing reactive power into the power system. Because of the presence of STATCOM devices in a fault loop, the voltage and current signals at relay point will be affected in both steady and transient state. This impact will affect the performance of exiting protection methods, such as distance relay.

This paper will analyze and explore the impact STATCOM employed in a transmission system on the Performance of distance relay. First, a detailed model of STATCOM is proposed and secondly, the analytical results based on symmetrical component transformation for single phase to ground fault on a transmission system employing STATCOM are presented, the simulation results clearly show the impact of STATCOM devices on the performance of distance relay.

\section{Mathematical Modeling of Statcom}

Static compensators or inverter circuit are mostly designed for voltage support of three phase balanced system. On transmission level, systems are normally balanced, but it is not the case during fault conditions of the distribution system. In three phase balanced system STATCOM produce a set of balanced reactive currents so that instantaneous power flow in STATCOM or from STATCOM is always zero.

Physically, compensator based on a three phase inverter circuit interconnects all the phases of thee phase ac system, providing full path for the exchange of reactive power between them. This type of exchange is disturbed during unbalanced operation of the ac system. In this case each phase of the inverter must provide unequal voltage. If PWM strategy of the control is used, it is possible to change phase voltages by changing control signals and keeping dc voltage constant. The value of dc reference voltage will determine the rating of the inverter and inverter will always draw some active power from the ac system to cover for its losses and keep the dc bus voltage constant.

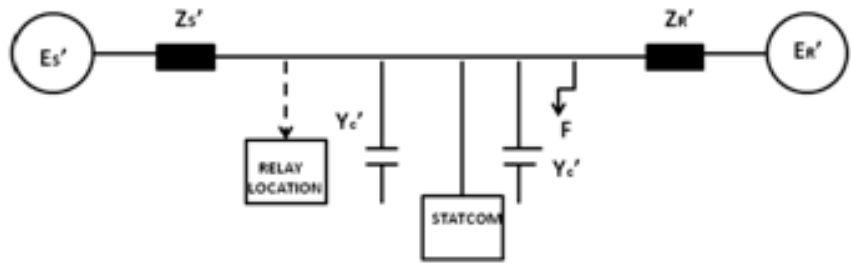

Fig. 1. Typical transmission line system

It is important to model the statcom and the relay individually and together before interfacing with the actual transmission system. This modelling helps to know about 
the characteristics of individual system. Hence consider a power system to model the STATCOM as shown.

The STATCOM is assumed to operate within its reactive power rating to maintain the voltage at its location under all operating conditions. In this study, the STATCOM is modelled [2] as a controlled-current-source that responds instantaneously to changing system condition

During a fault on the power system, the STATCOM is assumed to be in service as long as its reactive current does not exceed the STATCOM limits.

In this study, the STATCOM is rated to compensate the reactive power of the transmission line. The STATCOM current per-phase " $I_{\gamma}$ " is given by the following equation.

$$
I_{\gamma}=\left(\left(Z_{S}^{\prime}+\frac{Z_{L}}{2}\right)^{-1}\left(V_{\gamma}^{-1}\left(E_{S}^{\prime}+E_{R}^{\prime}\right)-2\right)-2 Y_{C}^{\prime}\right) V_{\gamma}
$$

The value of current " $I_{\gamma}$ " is automatically adjusted to changes in load angle " $\delta$," in such a way as to keep the midpoint voltage " $V_{\gamma}$ " constant.

where $Z_{s}$ '\& $Z_{r}$ ' are equivalent source impedance seen from the sending end and receiving end buses. $E_{s}{ }^{\prime} \& E_{r}$ ' are sending end and receiving end equivalent source voltages; $\mathrm{V} \gamma$ is midpoint voltage, $\mathrm{I} \gamma$ is compensator current

\section{MATHEMATICAL MODELING OF A RELAY}

For the analysis associated with the operation of a distance relay, the power system shown in Fig. 1 is used, the relay is installed on the right side of Bus S. The apparent impedance calculation [3] is based on symmetrical component transformation using power frequency components of voltage and current signals measured at relay point. It is assumed that signal acquisition, pre processing and sequence component calculations have been performed previously.

When a single phase to ground fault occurs at the ight side of STATCOM and the distance is $n^{*} L$ from the relay point, the positive, negative and zero sequence networks of the system during the fault can be shown as shown in fig 2 .

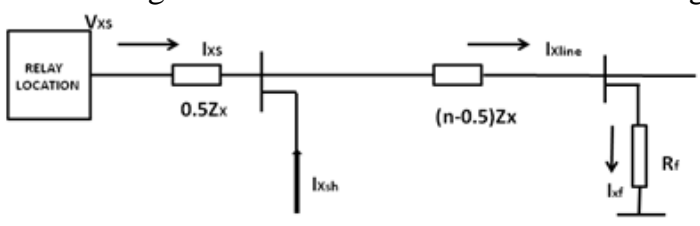

Fig. 2. The sequence network of a single phase fault

The same circuit will be considered for positive, negative and zero sequence networks. For the positive sequence network the notation ' $x$ ' equals to 1 , for negative sequence network it will be 2 , and for zero sequence network it is equal to 0 . Using these notations for the three sequence networks the following equations are obtained.

The sequence voltages at the relay point can be expresse as follows:

$$
\begin{gathered}
V_{1 s}=I_{1 s} 0.5 Z_{1}+I_{1 \text { line }}(n-0.5) Z_{1}+R_{f} I_{1 f} \\
V_{2 s}=I_{2 s} 0.5 Z_{1}+I_{2 \text { line }}(n-0.5) Z_{1}+R_{f} I_{2 f} \\
V_{0 s}=I_{0 s} 0.5 Z_{0}+I_{0 \text { line }}(n-0.5) Z_{0}+R_{f} I_{0 f}
\end{gathered}
$$

$$
\begin{gathered}
I_{1 \text { line }}=I_{1 s}+I_{1 s h} \\
I_{2 \text { line }}=I_{2 s}+I_{2 s h} \\
I_{\text {0line }}=I_{0 s}+I_{0 s h}
\end{gathered}
$$

where $V_{1 s} \quad V_{2 s}$ and $V_{0 s}$ are the sequence phase voltages at the relay location, $\mathrm{I}_{1 \mathrm{~s}} \mathrm{I}_{2 \mathrm{~s}}$ and $\mathrm{I}_{0 \mathrm{~s}}$ are the sequence phase currents at the relay location, $I_{1 \text { line }} I_{\text {2line }}$ and $I_{\text {oline }}$ are the sequence phase currents in the transmission line, $I_{1 f} I_{2 f}$ and $I_{0 f}$ are the sequence phase currents in the fault, $I_{1 s h}$ $I_{2 s h}$ and $I_{0 s h}$ are the shunt sequence phase currents injected by STATCOM, $Z_{1}$ and $Z_{0}$ are the sequence impedance of the transmission line , ' $n$ ' is the per unit distance of a fault from the relay location.

From above, the voltage at relay point can be derived as:

$$
\begin{aligned}
& V_{s}=n I_{s} Z_{1}+n I_{0 s}\left(Z_{0}-Z_{1}\right)+I_{s h}(n-0.5) Z_{1}+ \\
& 0.5) I_{s h 0}\left(Z_{0}-Z_{1}\right)+I_{f} R_{f}
\end{aligned}
$$

where

$$
\begin{gathered}
V_{s}=V_{1 s}+V_{2 s}+V_{0 s} \\
I_{s}=I_{1 s}+I_{2 s}+I_{0 s} \\
I_{s h}=I_{1 s h}+I_{2 s h}+I_{0 s h}
\end{gathered}
$$

Single phase to ground fault, the apparent impedance of distance relay can be calculated using the equation below

$$
Z=\frac{V_{R}}{I_{R}+\frac{Z_{0}-Z_{1}}{Z_{1}} I_{R 0}}=\frac{\mathrm{V}_{\mathrm{R}}}{\mathrm{I}_{\text {relay }}}
$$

where

$V_{R}, I_{R} \quad$ phase voltage and current at relay point

$I_{R 0} \quad$ zero sequence phase current

I relay The relaying current

If this traditional distance relay is applied to the transmission system with STATCOM, the apparent impedance seen by this relay can be expressed as:

$$
Z=n Z_{1}+\frac{I_{s h}}{I_{\text {relay }}}(n-0.5) Z_{1}+\frac{I_{f}}{I_{\text {relay }}} R_{f}
$$

It is clear from equation (13) that if only a solid single phase to ground fault is considered, the equation becomes:

$$
Z=n Z_{1}+\frac{I_{\text {sh }}}{I_{\text {relay }}}(n-0.5) Z_{1}
$$

The impact of STATCOM on the apparent impedance calculation for an impedance relay in a transmission line can be obtained using these equations.

\section{IV.Digital Distance Relay Algorithm}

Digital distance protection is a universal short-circuit protection. It's mode of operation is based on the measurement and evaluation of the short-circuit impedance, which is named by the algorithm of digital distance relay. This algorithm is used to input signals to DSP by discrete voltage and discrete current to judge whether faults occur or not. However, this method [4] is just a program. MATLAB has the advantage of conducting massive calculation functions and its program can be easily developed. 
Distance relays are also named impedance relays. They are used to calculate line impedance by measurement of voltages and currents on one single end. For example, for impedance type distance relays, the relays compare the setting impedance with the measurement impedance to determine if the fault is inside or outside the protected zone. They immediately release a trip signal when the impedance value is inside the zone 1 impedance circle of distance relay. For security protection consideration, the confirmation of a fault occurrence will not be made until successive trip signals are released in one season. Different formulas should be adopted when calculating the fault impedance due to different fault types.

Table I indicates calculation formula for all of the fault types [5]. Any three-phase faults can be detected from every formula in Table I. In order to reduce calculation burden, we design a fault detector and fault type selector. The fault detector can judge which fault type it is and then calculate fault impedance by selecting a suitable formula from Table I. If we don't use fault type judgment first, then the distance relay of programs must be calculated by all the six formulas in Table I at the same time, which causes much calculation burden.

TABLE I: FAULT IMPEDANCE CALCULATION ON DIFFERENCE FAULTS

\begin{tabular}{|l|c|}
\hline FAULT TYPE & FORMULA \\
\hline $\mathrm{AG}$ & $V_{A} /\left(I_{A}+3 c I_{O}\right)$ \\
\hline $\mathrm{BG}$ & $V_{B} /\left(I_{B}+3 c I_{O}\right)$ \\
\hline $\mathrm{CG}$ & $V_{C} /\left(I_{C}+3 c I_{O}\right)$ \\
\hline $\mathrm{AB}$ or $\mathrm{ABG}$ & $\left(V_{A}-V_{B}\right) /\left(I_{A}-I_{B}\right)$ \\
\hline $\mathrm{BC}$ or $\mathrm{BCG}$ & $\left(V_{B}-V_{C}\right) /\left(I_{B}-I_{C}\right)$ \\
\hline $\mathrm{CA}$ or $\mathrm{CAG}$ & $\left(V_{C}-V_{A}\right) /\left(I_{C}-I_{A}\right)$ \\
\hline
\end{tabular}

where $\mathrm{A}, \mathrm{B}$ and $\mathrm{C}$ indicates number of phase, $\mathrm{G}$ is ground fault , $\mathrm{V}$ and $\mathrm{I}$ are phasor of voltage and current, $\mathrm{c}=(\mathrm{Zo}-$ $\mathrm{Z1}) / \mathrm{Z1}$, Zo and Z1 are line of impedance zero-sequence, positive- sequence respectively. $I_{O}$ is zero-sequence current.

When the distance relays receive discrete voltage and current signal, it has to convert them to phasor. The Discrete Fourier Transform (DFT) is the most popular method to estimate fundamental phasors for digital relaying. The fullcycle DFT is described as following equation (15):

$$
\mathrm{X}=\frac{2}{N} \sum_{k=0}^{N-1} \mathrm{X}_{\mathrm{K}} \mathrm{e}^{-\mathrm{j} 2 \pi \mathrm{k} / \mathrm{N}}
$$

where $\mathrm{X}$ is complex phasor, $\mathrm{Xk}$ is the sample discrete data of the signal, and $\mathrm{N}$ is the number of samples per cycle. By using the above equation depending on the number of samples we can write the MATLAB program for conversion of discrete voltage and current signals into complex form.

In addition, when a fault occurs on transmission lines, the voltage and current signals are severely distorted. These signals may contain decaying dc components, subsystem frequency transients, high frequency oscillation quantities, and etc. The higher frequency components can be eliminated using low pass anti-aliasing filters with appropriate cut-off frequency, but the anti-aliasing filters cannot remove decaying dc components and reject low frequency components. This makes the phasors very difficult to be quickly estimated and affects the performance of digital relaying. Therefore, we usually use the mimic filter to removed the dc-offset components [6]. The mimic filter can be developed by digital method. Here, we want to pass the fundamental frequency signal $(50 \mathrm{~Hz})$ by the filter. Then, assuming the gain $\mathrm{L}$ equals 1 and the samples frequency is, $f_{S}\left(f_{S}=1 / T_{S} \quad\right)$ finally, we obtain a formula (16).

where

$$
\left|L\left(1+\tau f_{S}\right)-L \tau f_{S} \cos \omega T_{S}+j L \tau f_{S} \sin \omega T_{S}\right|=1
$$

$\omega=2 \times \pi \times 60, \tau$ is time constant for user definition.

To remove the dc-offset components, MATLAB program can be developed using the above equation.

Hence from the equations (15) \& (16) we can prepare the digital distance relay algorithm for filtering the waveforms and converting them into phasors. The algorithm is presented as follows.

Step 1: Get the data of voltage and current signals from simulation of system under study which are in discrete form.

Step 2: Apply the filtering technique for those current and voltage signals to remove dc-offset components. Apply the same filtering technique for all the three phase current and voltage signals.

Step 3: Phasor estimation using DFT.

Step 4: Calculate the impedance values for each phase under fault conditions using the formulas tabulated in table 1.

Step 5: Draw the impedance trajectory.

As shown in the above algorithm the currents and voltages are send through the mimic filter in order to remove the dc-offset components and for the phasor estimation. Later using these values we can find out the impedance values for all the phases in faulty conditions. From the above discussion, the MATLAB can easily finish all of algorithms for protective relays. With the advantage that SIMULINK can easily simulate power system faults, the design and the test of protective relays can be achieved with ease. Its major characteristic of integrating system fault simulation and protection relay algorithms in a software system can enhance the efficiency of protection relay test.

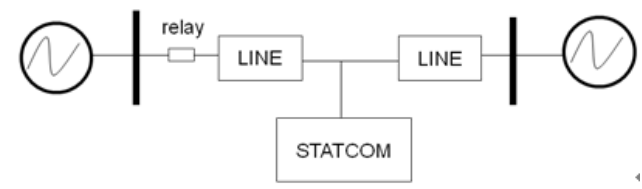

Fig. 3. One-line diagram of simulation system

\section{Simulation OF POWER SYSTEM}

With the updated versions of MATLAB/SIMULINK, the model development of power system components is onward to perfection. Due to the fast development of new technologies, which improve the power transfer efficiency and the optimum utilization of system capability, power electronic equipment like TCSC - UPFC - STATCOM...and so on may be widely used in power systems In the future. Thus, the selection and the setting of protective relay should 
be evaluated and tested thoroughly [7]. Here, SIMULINK includes variant basic power components, which can be used alone or in combinative use to finish all kinds of power system network simulations.

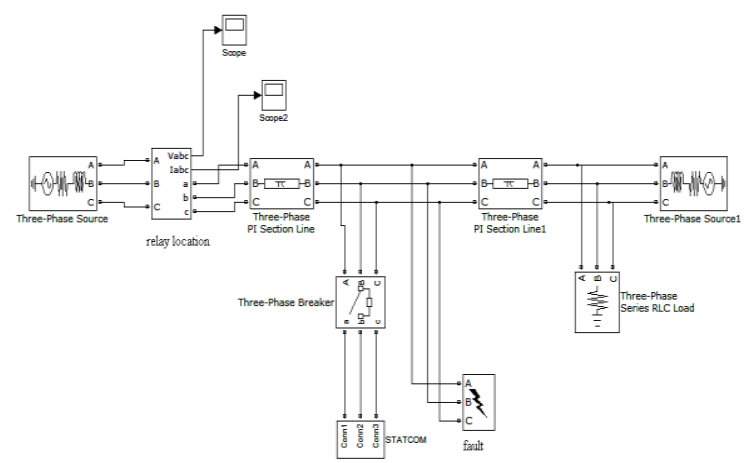

Fig. 4. SIMULINK diagram for the power system

As shown in the SIMULINK diagram the STATCOM was designed for the given requirements, i.e. 3-level, 12pulse inverter was built in MATLAB and simulation is individually for this STATCOM to ensure that the output is 3-level output and then it will be made as a subsystem and given to the power system under study.

In addition, SIMULINK provides some options like realtime display, storing data in WORKSPACE and hard disk after the signals data is released by filter. As shown in Fig.4, we capture signals and store them in WORKSPACE from the simulation systems, which are provided for using input of distance relay algorithm.

For the voltage and current waveforms obtained from the simulation of Fig 4, the digital distance relay algorithm is applied. And by using impedance calculation formulas from table 1, the impedance trace can be plotted for all the six faults given in table. And the simulation results can be traced for all the faults. Here, the simulation results for the system with and without STATCOM for phase A fault are presented as an example.

\section{SIMULATION RESULTS}

Here the simuation results are presented for phase A fault with \& without STATCOM for an impedance relay. From the results, the system will be out of the faulty region with the presence of STATCOM as shown. The results are obtained using the formulas in Table I for impedance calculation. The results for the remaining faults can also be obtained using the formulas given.

The waveforms given below are impedance trajectory for healthy system, impedance trace without STATCOM, and impedance trace with STATCOM for phase A fault.

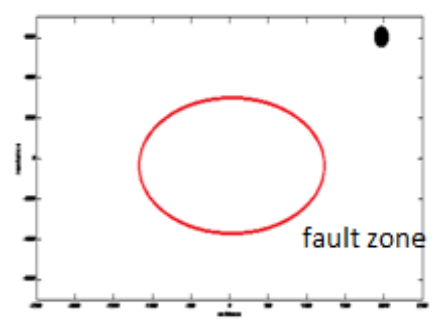

Fig. 5. Impedance trajectory for healthy system

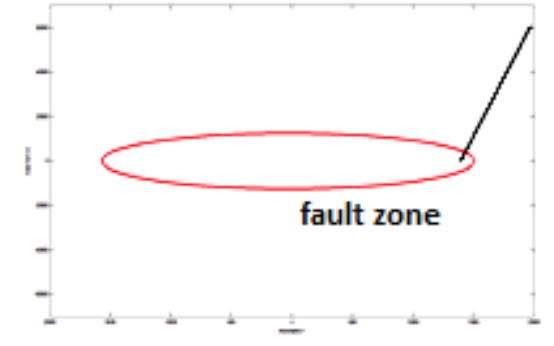

Fig. 6. Impedance trace for phase A fault without STATCOM

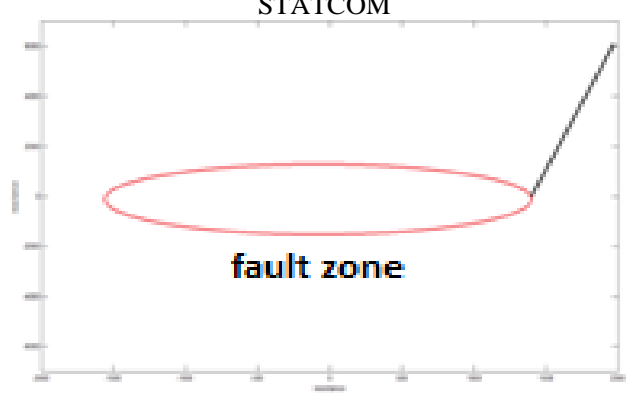

Fig. 7. Impedance trace for phase A fault with STATCOM

\section{CONCLUSION}

In this paper the modelling of impedance relay with STATCOM is presented Using digital distance relay algorithm. And the results are presented with the MATLAB simulation for different faults. The same can be done with different FACTS devices also. From the simulation results, STATCOM can reduce the fault impedance by distorting the voltage and current waveforms due to the injection of reactive power into the system.

\section{APPENDIX A (Test System DATA)}

Equivalent source impedances at sending end and receiving ends:

$$
Z_{s}=(21.44+3.214 \mathrm{j}) \Omega, Z_{R}=(17.5+1.309 \mathrm{j}) \Omega
$$

Line constant:

$$
\begin{array}{cl}
R_{O}=0.275 \Omega, & L_{O}=3.725 H, C_{O}=6.71 \mathrm{nF} \\
R_{1}=0.0275 \Omega, & L_{1}=1.345 H, C_{1}=9.483 n F
\end{array}
$$

STATCOM: Rating: 160MVA Transformer: $15 \mathrm{kv} / 130 \mathrm{kv}$

LOAD: Rating : 138KV Active power : 10MW

\section{REFERENCES:}

[1] The Math Works, Inc, "Using MATLAB," 1999.

[2] K. E. Arroudi, G. Joos, and D. T. M. Gillis, "'Operation of Impedance Protection Relays With the STATCOM," IEEE Trans. on Power Delivery, vol. 17, no. 2, April 2002, pp. 381-387

[3] X. Y. Zhou, H. F. Wang, R. K. Aggarwal, and P. Beaumont, "The Impact of Statcom On Distance Relay" $15^{\text {th }}$ PSCC, Liege, pp. 22-26 2005.

[4] L. C. Wu and C. W. Liu, "Modeling and Testing of a Digital Distance Relay Using MATLAB/ SIMULINK,"IEEE, 2005.

[5] T. S. Sidhu, D. S. Ghotra, and M. S. Sachdev, "A Fast Distance Relay Using Adaptive Data Window Filters," IEEE/PES Summer Meeting, July 2000, pp.1407-1412.

[6] G. Benmouyal, "Removal of Decaying DC in Current Waveforms Using Digital Mimic Filtering," IEEE Trans. on Power Delivery, vol. 10, no. 2, April 1995, pp. 621-630. 